\title{
MINORITY INFLUENCE IN OPINION SPREADING
}

\author{
Ugo Merlone \\ Department of Psychology \\ University of Turin \\ Via Verdi 10 \\ Torino, TO 10124, ITALY \\ Davide Radi \\ Department of Management \\ Università Politecnica delle Marche \\ P.zza Roma 22 \\ Ancona, AN 60121, ITALY
}

\author{
Angelo Romano \\ Department of Psychology \\ Unversity of Turin \\ Via Verdi 10 \\ Torino, TO 10124, ITALY
}

\begin{abstract}
Social influence has been an object of interest of social psychology for a long time. More recently, sociophysics and Galam's model provide an explanation of rumors spreading in a population explaining some interesting social phenomena as diffusion of false information. Although Galam's model and its recent formalizations are suitable to describe some social behavior, they take into account populations with homogeneous agents. Some recent contributions consider agents who do not change opinion and in some cases are able to persuade the others. Starting from social psychology studies about the role of specific seat occupation we provide a heterogeneous model in which those holding minority opinions can strategically choose specific social gatherings to exert their influence. We simulate the opinion dynamics comparing situations in which there is the minority to others with homogeneous agents. Our results show how the opinion dynamics is affected by the presence of such a minority.
\end{abstract}

\section{INTRODUCTION}

Social psychology has widely investigated the role of social context in shaping individuals' opinions, attitude and decision-making (Brown 1988, Wood, Lundgren, Ouellette, Busceme, and Blackstone 1994). Social influence, a research area in social psychology, (Brown 1988), shows how consensus arises among individuals, explains how minority positions may succeed or fail in their diffusion, and sheds light on the relation between cognitive processes underlying majority and minority influence (Moscovici and Personnaz 1980).

Interesting seminal studies in this area demonstrated the importance of majority influence over individuals (Asch 1956, Festinger 1954). For example, in one of the most famous experiments conducted by Asch (1956) individuals were introduced to a task on visual discrimination. The instructions consisted in matching the length of a given line with other three. Participants were put in a group which was actually composed by confederates and had to express their judgment after having heard others' opinions. The confederates gave the wrong answer on 12 trails over a total of 18. The findings revealed that even when the perceptual judgments of confederates were objectively biased, individuals tended to conform to the majority. 


\section{Merlone, Radi, and Romano}

By contrast, other contributions (Moscovici, Lage, and Naffrechoux 1969, Moscovici and Personnaz 1980) analyzed the effect of minority influence on individual decision-making. These studies have been groundbreaking and helped to understand the diffusion of innovation (Mugny and Papastamou 1980), the analysis of social change in social systems (Mugny and Papastamou 1982), and how minority may influence dominant positions (Paichler 1976).

The experiment conducted by Moscovici, Lage, and Naffrechoux (1969) had the same structure of Asch's experiments but in this case the confederates represented the minority of the group. As in Asch experiments the task was based on perception: individuals had to judge the color of several slides, blue or green. In six-subject groups, four individuals were the experimental subjects while two subjects were actually confederates. From their analysis Moscovici, Lage, and Naffrechoux (1969) found that when minority had a coherent behavior was able to change about $8 \%$ of total answers when compared to the control condition with no confederates.

The studies on minority are quite interesting to social psychologists as minority influence has a deep impact on individuals' beliefs (Wood, Lundgren, Ouellette, Busceme, and Blackstone 1994): in fact, in Moscovici, Lage, and Naffrechoux (1969), the authors revealed that biased perception induced by minority influence was influencing judgment also after the first task. Moscovici and Lage (1976) compared the effects triggered by minority and by majority and confirmed the hypothesis that majority influence affects manifest level, that is just the public opinion showed to others, while minority influences both public and private changes (Moscovici and Personnaz 1980). Accordingly, the change promoted by majority is called compliance, whereas the change promoted by minority, conversion (Maass and Clark 1984).

From the seminal experiments of Moscovici (Moscovici and Personnaz 1980, Moscovici, Lage, and Naffrechoux 1969) there have been several studies providing new insights and analysis on the effect of minority (Butera, Mugny, Legrenzi, and Perez 1996, Shuper and Sorrentino 2004). Reviews on the main processes underlying minority influences can be find in Crano and Seyranian (2007), Wood, Lundgren, Ouellette, Busceme, and Blackstone (1994), and Maass and Clark (1984). In their review on 15 years of research on minority influence Maass and Clark (1984) argued that a certain degree of flexibility of minority may increase its effect on other respondants (Mugny 1975). Baker and Petty (1994) analyzed the differences in individual scrutiny when a position is endorsed by a majority or a minority, Butera, Mugny, Legrenzi, and Perez (1996) investigated the use of inductive reasoning according to whether the source of information come from minority or majority and Shuper and Sorrentino (2004) studied individual differences in certainty orientation in being influenced by a minority.

Studies on minority influence are interesting not only for the analysis of the underlying psychological processes but, as underlined in the review of Maass and Clark (1984), find several examples of applications as the analysis of the role of women in French society (Paichler 1976); decision-making (Nemeth and Wachtler 1974), and air pollution (Mugny and Papastamou 1980).

\section{OPINION SPREADING}

Although experimental studies in psychology have provided interesting insights on the role minority influence, this kind of analysis presents some limits. For example, as discussed in Moscovici and Personnaz (1980), there is the possibility that minority influence has long-term effects and it is almost impossible to take into account this temporal dimension in these experiments. Accordingly, in this case it is necessary to implement some methods aiming at providing and test the hypothesis and theories derived in this context in order to reinforce and better understand the phenomenon. An example of the integration can be found in Galam and Moscovici (1991): in this paper the authors combine a number of social psychology hypothesis with the methods of statistical physics. In particular, in this work the authors try to model collective phenomena taking into account the studies on polarization of attitudes and decisions. According to the Authors, this integration may promote the understanding of emergent phenomenona as well as a context to validate these concepts. 


\section{Merlone, Radi, and Romano}

Consensus within group has also been analyzed by mathematical modeling, as for example in DeGroot (1974) and Berger (1981). These kind of approaches allow to understand processes and factors important to reach consensus in groups. In the last years, the interplay between strategic interaction and social influence has been investigated in order to study the possibility of consensus equilibria (Buechel, Hellmann, and Klößner 2013). For example, in this kind of models (Buechel, Hellmann, and Klößner 2013), agents can change their opinion in order to follow others' opinions. This is explained by the fact that individuals would incur in utility losses if anchored to the own reasons. A different line of research when studying opinion dynamics is proposed in (Hegselmann and Krause 2002), and, more recently, by (Hegselmann, König, Kurz, Niemann, and Rambau 2014). Another example of this interplay can be seen in Buechel, Hellmann, and Pichler (2012), in which starting from the pioneeristic DeGroot model of opinion there is an analysis of the influence of cultural traits in group decisions (DeGroot 1974).

\subsection{Galam's model}

Several other contributions on the analysis of processes of social influence have been provided by other disciplines. For example sociophysics, using tools and concepts from the physics of disordered matter, is making a serious attempt to describe some aspects of social and political behavior; see for example, Galam, Gefen, and Shapir (1982), Stauffer (2012), Nyczka and Sznajd-Weron (2013) or, for a critical review, Galam (2012).

In particular Galam (2003) proposes a model to understand how rumors can be propagated within a population. In his models, at time $0, N$ individuals hold an opinion-either True or False-on a particular subject and discuss the issue at different social meetings. In the course of time $t=1,2, \ldots$, the same people will meet again and again randomly in the same group configurations and may change their opinion according to a majority rule.

According to the model, although an opinion can be the minority at the beginning, after a series of social gatherings there could be a significant spread. This kind of formalization fits with the phenomenon of diffusion of rumors in public debates. For example, in Galam (2003), the Author deals with the spread of the notice in which it was claimed that no plane was crashed in the Pentagon on September 11.

The formalization of Galam's model considers a $N$ person finite population and assumes that only two opinions, '+' and '-', are possible. The social space consist of several tables where the discussion takes place. From this initial configuration, people start discussing the issue at the first social meeting. Each new cycle of multi-size discussions is marked by an unitary time increment. In a group of size $k$ with $j$ agents sharing opinion ' + ' and $k-j$ sharing opinion ' - ', all $k$ members adopt opinion ' + ' if $j>k / 2$; on the contrary, everybody adopts opinion '-' if $j<k / 2$; in the symmetric case $j=k / 2$ the outcome is determined assuming a bias in favor of one of the two opinions; these rules are called discussion functions. In the example depicted in Figure 1 there are 24 individuals and the social space consist of 5 tables with sizes 3, 4, 4, 5 and 8 respectively. For the sake of brevity social spaces are denoted by a vector in which components are the table sizes, in Figure 1 we have $\mathbf{n}=(3,4,4,5,8)$

Therefore, the set of possible states of the population with respect to opinion ' + ' is $S=\{0,1, \ldots, N\}$, where state 0 means consensus has been reached on opinion '-' while, on the contrary, state $N$ means consensus has been reached on opinion ' + '. In other words, the state of the population is the number of individuals holding opinion ' + '. Not all the states in $S$ are necessarily feasible. State feasibility depends obviously on both social space and the discussion function which is adopted.

Galam (2003) uses the binomial distribution to approximate the probability of having a specific number of ' + ' opinions at each table. As long as populations with a large number of agents and small size discussion groups are considered the use of the binomial distribution is a good modeling approximation. However this approach may create distortions in the prediction of the dynamics of rumors in small communities. In order to deal with this problem, Merlone and Radi (2014) formalize the process as an absorbing Markov chain, in which the states of the process correspond to the number of agents holding one opinion and compute 


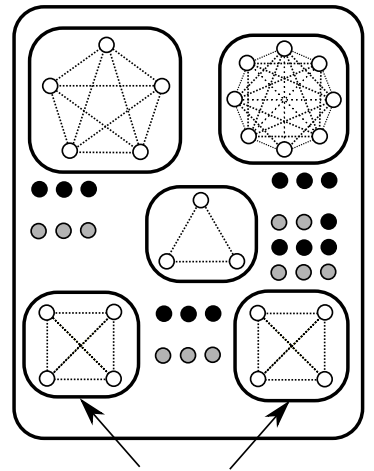

Empty social spaces

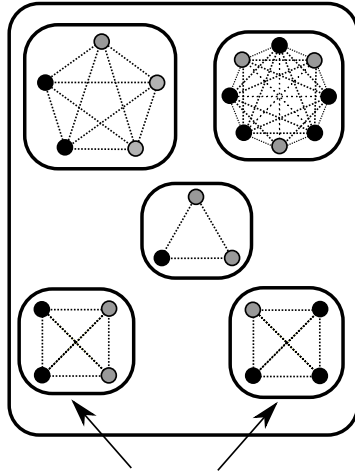

Social gathering before discussion

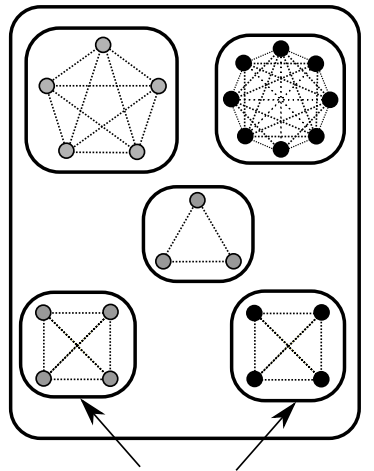

Social gathering after discussion

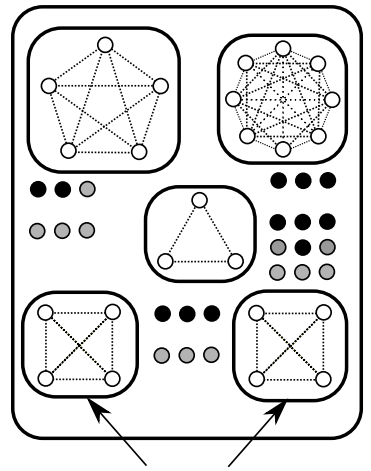

Empty social spaces

Figure 1: A one step opinion dynamics. First stage, people sharing the two opinions are moving around. Grey have opinion '-' while black have opinion '+'. No discussion is occurring with 11 gray and 13 black. Second stage right, people take place at the tables the social space consists of. Third stage, within each group consensus has been reached. As a result, they are now 12 gray and 12 black. Last stage, people are again moving around with no discussion.

the transition matrices for several examples. The transition matrix is a stochastic matrix describing the probabilities of particular transitions across the state space (Behrends 2000).

However both Galam's original model and its recent formalizations (Merlone and Radi 2014) consider only a majority rule with homogeneous agents. In the following section we introduce strategic minority agents in order to study how minority can influence opinion dynamics. We consider a strategic minority able to sit always at the maximum size table in which it represents the majority and then can exert its influence. Other contributions have considered agents using influence. For example, in Galam's model Ellero, Fasano, and Sorato (2013) introduce opinion leaders, i.e., agents who always have opinion ' + ' and are able to persuade all the agents at their table. More generally, when interacting in networks, West, Turalska, and Grigolini (2014) consider committed minority agents keeping an assigned decision independently of the opinion of their neighbors. By contrast, the agents we consider here have some strategic abilities to find the best table where to exercise their influence at the cost of potentially being influenced.

\section{MODELING MINORITY}

When modeling minority we can consider several different behaviors, some of which can be the result of complex strategies, communication and coordination. In Galam (2003), one of the fundamental characteristics of the model is the presence of a threshold to reach consensus: if minority is smaller than this threshold, then its effect on opinion is irrelevant. Nevertheless, this does not capture the cases in which a minority may adopt successful strategies in order to reach consensus.

In this paper, we try to fill this gap by considering a minority able to exert its influence in strategic tables in order to control opinion dynamics, for this reason it will be called strategic minority. In our opinion, this approach is closer to the psychological theories on minority influence (Wood, Lundgren, Ouellette, Busceme, and Blackstone 1994) focusing on a minority able to influence others with coherent strategies and may provide some suggestions to understand real cases in which such a strategy is followed by a limited number of agents.

In particular, in our model first the strategic minority agents will all sit together at one of the tables where they can exert an influence, then other agents are randomly distributed among tables, as in the original model. This seating strategy relates to some well known studies in which seating at particular places in tables is related to influence, see Sommer (1961), Hare and Bales (1963), Strodtbeck and Hook (1961). 


\section{Merlone, Radi, and Romano}

We run two different types of simulations: one replicating Galam's findings (Galam 2003) considering two of the examples used in Merlone and Radi (2014). As discussed previously, the social influence of minority is modeled as the capacity of a strategic minority of choosing always the best social configuration to exert influence on others' behavior. In other words, the majority influence rule remains the same as in the Galam studies, but in our minority modeling the agents representing the minority have the ability to find, when possible, a social gathering where they can influence the others. Furthermore, our strategic minority is more fragile than the opinion leaders considered in Ellero, Fasano, and Sorato (2013) as the latter always have opinion ' + ' and are able to persuade all the agents at their table.

\section{RESULTS}

The approach presented by Merlone and Radi (2014) considers homogeneous agents and is based on analytical derivations. By contrast, with strategic minority, agents heterogeneity makes such an explicit computation of the transition probabilities too difficult and we resort to simulation. In particular we consider three different social spaces. First we analyze two of the examples considered in Merlone and Radi (2014) in order to compare how our simulation results match the analytical results there provided. Both examples consist of 10 agents, yet with two different social spaces: $\mathbf{n}_{1}=(3,3,4), \mathbf{n}_{2}=(5,5)$. Finally, we consider how strategic minority may affect the opinion dynamics in the example depicted in Figure 1, i.e., 24 agents $\mathbf{n}_{3}=\mathbf{n}=(3,3,4,5,8)$. We select this specific example to show the dynamics in a more complex -yet still manageable- social space.

For each social space we compare two scenarios: one with homogeneous agents and one with a strategic minority. For each scenario 400,000 simulations were run to determine the transition matrix probability. In the following, $M$ will denote the transition matrices probability of homogeneous agents while $\hat{M}$ will denote the transition matrices with a strategic minority. From these matrices we compute the probability to converge to the absorbing states; they are represented graphically together with the expected time of convergence to the absorbing states. These graphical representations are provided in Figures 2 to 7 . The left side of these figures represents the probabilities to converge, starting from state $j \in S$, to the respective absorbing states for each social space and agents population. The right side of the figures represents the expected time, $z_{j}$, of convergence to absorbing states for each initial state $j \in S$. As we are considering discrete states the dashed lines are there for illustrative purpose only. Obviously, when $j$ is larger than $N / 2$, opinion ' + ' is no longer a minority opinion. However the strategic minority agents' behavior may still have consequences on the opinion dynamics.

\subsection{The case of 10 agents}

As mentioned above, in Merlone and Radi (2014) two 10-agent social spaces are considered and the relative transition matrices are analytically derived and reported. In order to validate the model (Sargent 1984), we consider the same 10-agent social spaces in order to compare the probability transition matrices we find by simulation.

First we consider the two 10-agent social spaces analyzed in Merlone and Radi (2014) to compare the probability transition matrix obtained by simulation to those analytically derived with two different social spaces: $\mathbf{n}_{1}=(3,3,4), \mathbf{n}_{2}=(5,5)$. The transition matrices of the two social spaces considering Galam's model and strategic minority influence are reported in transition matrices (1) and (2) respectively. As proved in Merlone and Radi (2014), when considering social space $\mathbf{n}_{1}=(3,3,4)$ the feasible states are 0 , $3,4,6,7,10$, while with social space $\mathbf{n}_{2}=(5,5)$ feasible states are $0,5,10$.

State 0 represents the situation in which all the population converge to the opinion '-', while 10 is the state in which all agents converge to the opinion ' + '.

$M_{\mathbf{n}_{1}}$ and $M_{\mathbf{n}_{2}}$ are consistent with Galam's model prediction and the findings of Merlone and Radi (2014). 


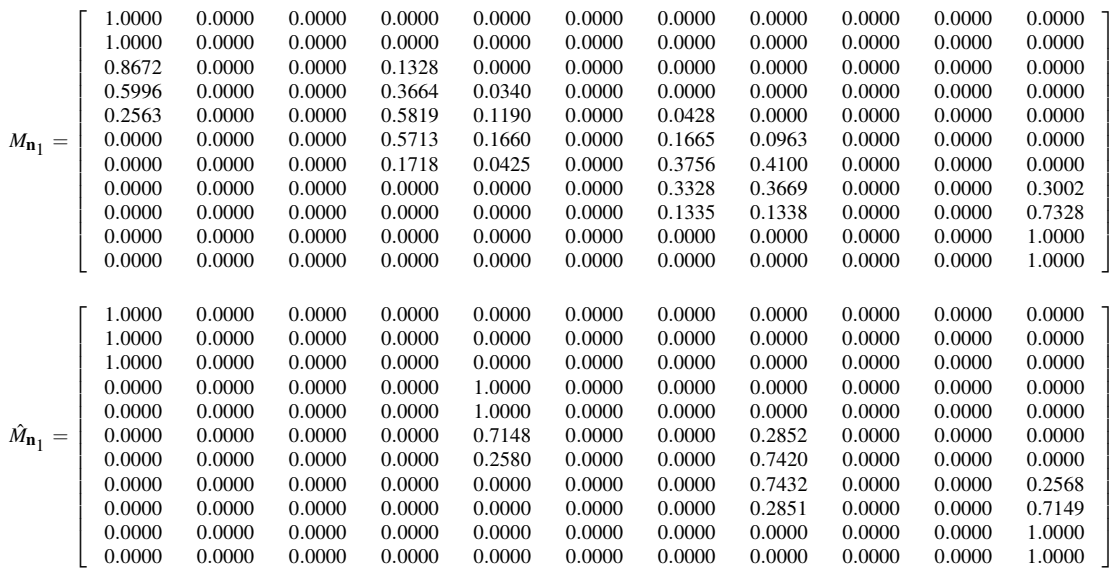

$\hat{M}_{\mathbf{n}_{1}}$ and $\hat{M}_{\mathbf{n}_{2}}$ represent the transition matrices when considering a minority which always seats in a fixed table in which the minority have a majority influence. As we can observe, we have in both cases three absorbing states.

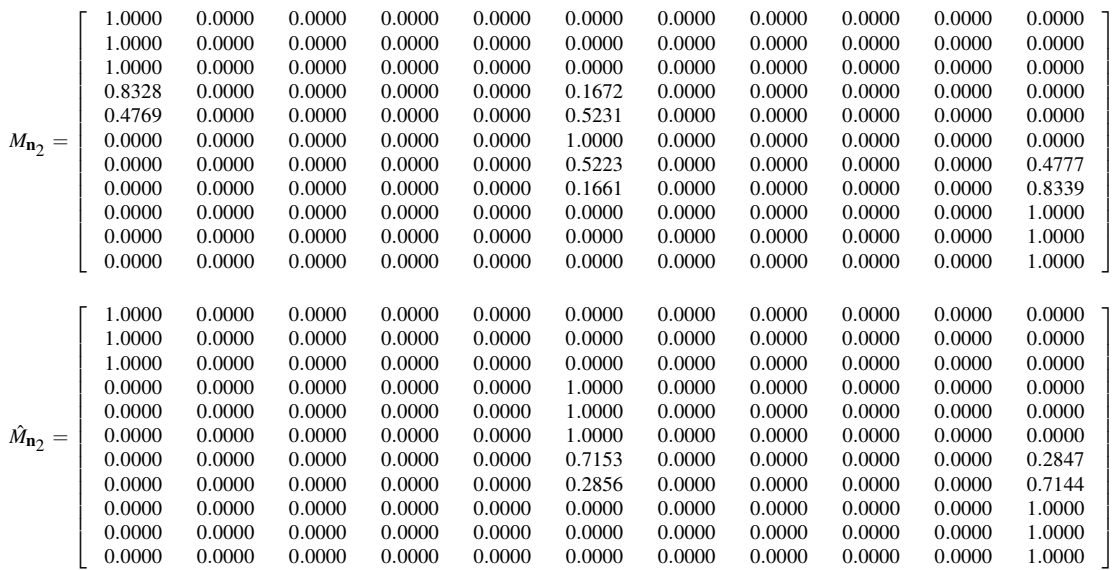

As it concerns social space $\mathbf{n}_{1}$, by comparing Figures 2(a) to 3(a) we can see that, with strategic minority agents, state 4 becomes absorbing; this is confirmed by the entries in the fourth column of probability transition matrix (2) and also in Figure 3(b) where the expected number of steps to converge to state 4 is 0.

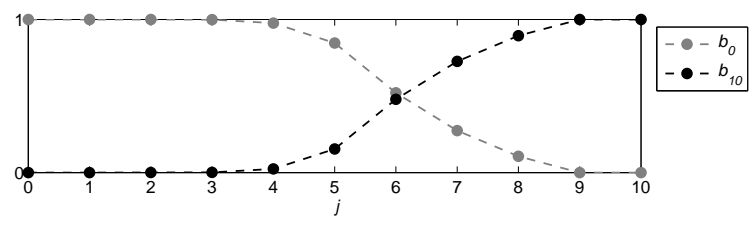

(a)

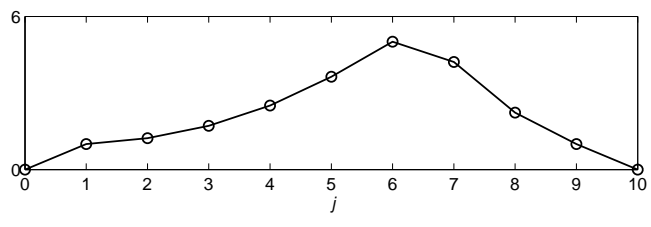

(b)

Figure 2: Case $\mathbf{n}_{1}=(3,3,4)$ without minority. Depending on the initial state $j \in S$ : (a) probability to converge to the absorbing states 0 (grey circles) and 10 (black circles); b) expected time of convergence to consensus (either 0 or 10).

When considering social space $\mathbf{n}_{2}$, besides states 0 and 10 also state 5 is absorbing (see Merlone and Radi 2014). In this case the effect of minority is more subtle as states 4,5 and 6 are a closed set, namely 


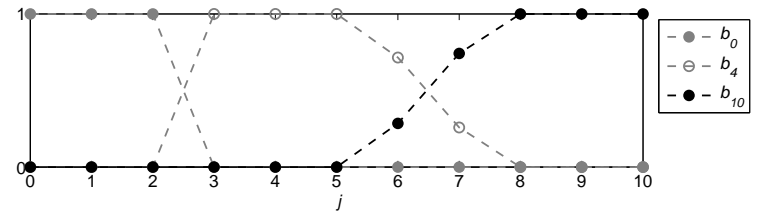

(a)

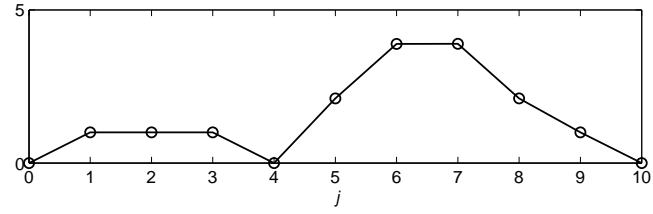

(b)

Figure 3: Case $\mathbf{n}_{1}=(3,3,4)$ with a 3 minority. Depending on the initial state $j \in S$ : (a) probability to converge to the absorbing states 0 (grey circles), 4 (hollow circles) and 10 (black circles); b) expected number of steps to converge to absorbing states 0,4 or 10 .

starting from each one of these three states, the probability to reach a state different from these three states is zero ${ }^{1}$. Moreover, such a closed set is such that with probability 1 the process reaches the absorbing state 5 in one step. The strategic minority does not affect the number of steps to convergence as it can be seen by comparing Figures 4(b) and 5(b).

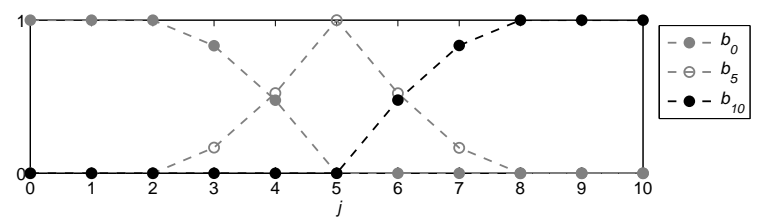

(a)

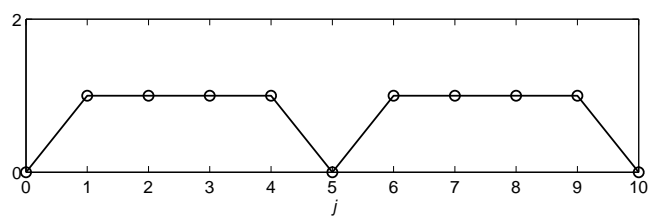

(b)

Figure 4: Case $\mathbf{n}_{2}=(5,5)$ without minority. Depending on the initial state $j \in S$ : (a) probability to converge to the absorbing states 0 (grey circles), 5 (hollow circles) and 10 (black circles); b) expected number of steps to converge to absorbing states 0,5 or 10 .

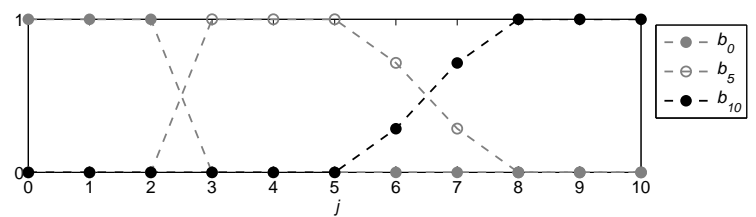

(a)

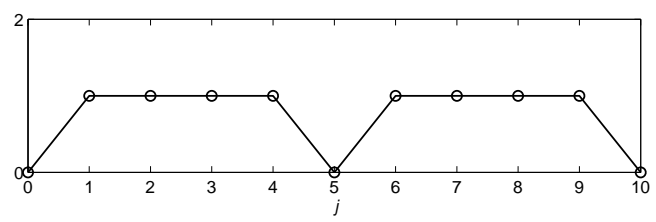

(b)

Figure 5: Case $\mathbf{n}_{2}=(5,5)$ with a 3 minority. Depending on the initial state $j \in S$ : (a) probability to converge to the absorbing states 0 (grey circles), 5 (hollow circles) and 10 (black circles); b) expected number of steps to converge to absorbing states 0,5 or 10 .

Comparing the last column of matrices $M_{\mathbf{n}_{1}}$ and $M_{\mathbf{n}_{2}}$ to respectively $\hat{M}_{\mathbf{n}_{1}}$ and $\hat{M}_{\mathbf{n}_{2}}$ we can see that the entries of transition matrices with strategic minority are not larger than the corresponding ones with no strategic minority. This is because when the number of agents with opinion ' + ' is large, the strategic

\footnotetext{
${ }^{1}$ For further details see Behrends (2000). A closed set can also be called closed communicating class (Ibe 2009)
} 


\section{Merlone, Radi, and Romano}

positioning of minority agents is counterproductive. In facts, in this case strategic agents would be more effective when spreading around.

\subsection{The case of 24 agents}

Now consider the case of 24 agents with social space: $\mathbf{n}_{3}=\mathbf{n}=(3,3,4,5,8)$ as illustrated in Figure 1 . In this case, the feasible states are $0,3,4,5,7,8,9,11,12,13,15,16,17,19,20,21$, and 24. Transition matrix (6) for strategic minority shows interesting results as in this case we do not have a new absorbing state. In fact, when considering column 6 of transition matrix entries on rows 4 and 5 are 1.00 as expected. Indeed, when agents starting with opinion ' + ' are just 3 or 4 they are unable to obtain more than 5 agents with opinion ' + '. By contrast, on row 6 of the same column we find 0.99 as, when there are 5 agents with opinion ' + ', the strategic minority agents will seat at the size 5 table and there is a positive probability that the other two ' + ' opinions agent will seat at the size 3 table; in such a case, at the following turn, there will be 8 agents with opinion ' + '.

In (Galam 2003) the killing point is defined as the point where the monotonic flow -towards one of two stable fixed points- changes direction. The killing point is important to characterize the rumor dynamics when as it determines the border of the basin of attractions of the absorbing states. As with social space $\mathbf{n}_{3}$ the absorbing states are consensus either on '-' or ' + ', this notion has clear implications. Although the killing point can be formalized either as in the original Galam model (Ellero, Fasano, and Sorato 2013) or in the Markov chain formalization (Merlone and Radi 2014), intuitively it can be seen as the intersection between the two dashed lines in Figures 5(a) and 6(a).

In Figure 6(a) we can see that absorbing state 0 is prevalent as long as the initial number of agents with opinion ' + ' is smaller than 14 , that is, the killing point is between 14 and 15 . By contrast, with a strategic minority (Figure 7(a)) the killing point is between 2 and 3 and the probability to converge to ' + ' is one when at the beginning more than 2 agents hold this opinion. Nevertheless, convergence is quite slow as illustrated in Figure 7(b). This is because when starting with 3 strategic minority agents starting with opinion ' + ' they will seat at the 5 size table and at the following turn there will be always 5 agents with opinion ' + '; therefore the probability to end in the absorbing state 24 is small but positive.

In conclusion, although the strategic minority is able to drive consensus on opinion ' + ' there is a steep price to pay in terms of time to converge to such a consensus, when the initial number of agents with opinion ' + ' is smaller than the killing point for social space $\mathbf{n}_{3}$ with no strategic minority agents.

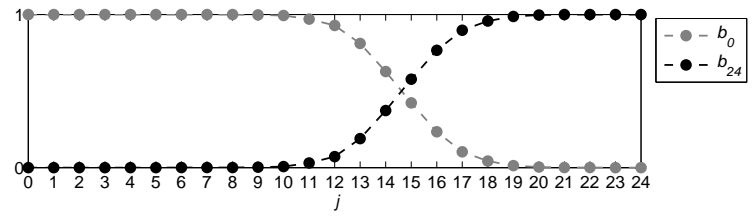

(a)

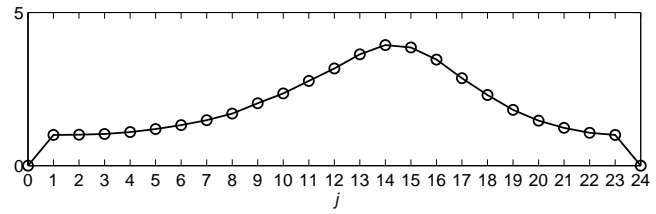

(b)

Figure 6: Case $\mathbf{n}_{3}=(3,4,4,5,8)$ without minority. Depending on the initial state $j \in S$ : (a) probability to converge to the absorbing states 0 (grey circles) and 24 (black circles); (b) expected number of steps to converge to consensus (either 0 or 24 ).

By comparing the last column of matrices $M_{\mathbf{n}_{3}}$ and $\hat{M}_{\mathbf{n}_{3}}$, we can notice that the probability to be in the state $N$ seems smaller without the strategic minority. In other words, we can see that also for this social space, when the number of agents with opinion ' + ' is large the strategic positioning of minority agents is counterproductive. 


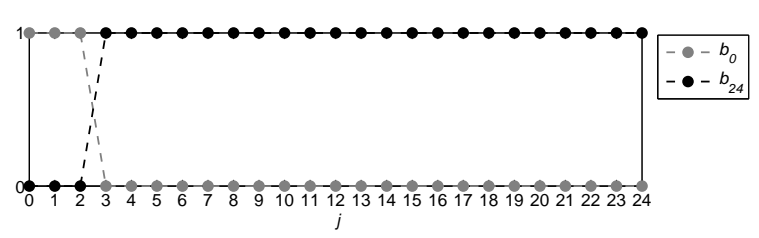

(a)

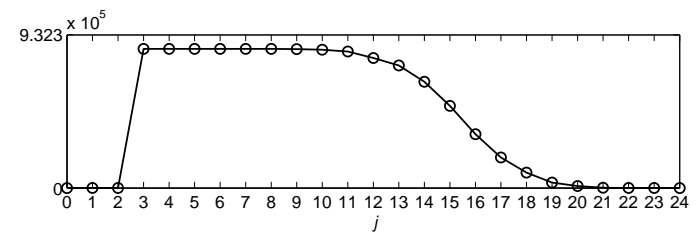

(b)

Figure 7: Case $\mathbf{n}_{3}=(3,4,4,5,8)$ with a 3 minority. Depending on the initial state $j \in S$ : (a) probability to converge to the absorbing states 0 (grey circles) and 24 (black circles); b) expected number of steps to converge to consensus (either 0 or 24 ).

\section{CONCLUSIONS}

In this paper, we analyzed minority influence on opinion dynamics. In particular, we considered a minority able to interact in social gatherings by taking place at strategic positions. The particular strategy we consider may be effective in situations in which minority size is small and the risk to be wiped out by the majority is high. Furthermore, this strategy is related to some well known studies in which seating at particular places in tables is related to influence, see Sommer (1961), Hare and Bales (1963), Strodtbeck and Hook (1961).

By simulation we found the transition matrices for the different social space we considered and were able to observe the effects of strategic minority in terms of transition probabilities.

The presence of a strategic minority affects the opinion dynamics in several ways. Firstly, absorbing states different from opinion consensus appear; this is interesting because in the original model (Galam 2003) only consensus to either opinion could be observed. By contrast, with a strategic minority the dynamics is less predictable and under some conditions disagreement remains. Secondly, although the strategic minority can tip the balance in favor of one opinion there is a cost to pay for this. In fact, the very same strategy this kind of minority adopts may slow reaching consensus on the opinion they support.

Future research will focus on analyzing different social spaces. In particular, it will be interesting to introduce minority influence in opinion dynamics when considering networks (Merlone, Romano, and Radi ). Finally, it will be also interesting to analyze the opinion dynamics when considering strategies different from the the one considered in this paper. 

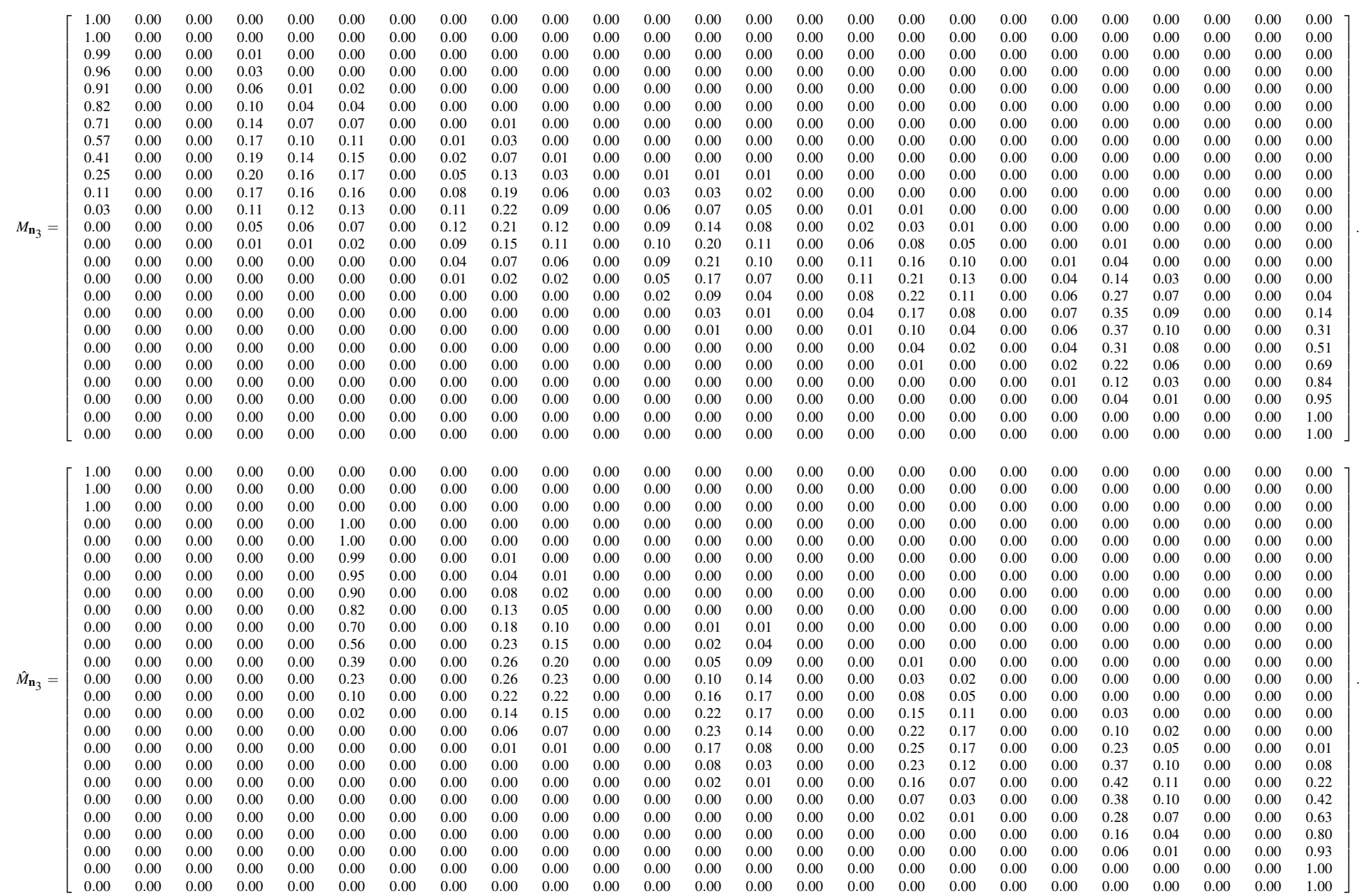


\section{REFERENCES}

Asch, S. E. 1956. "Studies of independence and conformity: I. A minority of one against a unanimous majority". Psychological monographs: General and applied 70 (9): 1-70.

Baker, S. M., and R. E. Petty. 1994. "Majority and minority influence: source-position imbalance as a determinant of message scrutiny". Journal of Personality and Social Psychology 67 (1): 5.

Behrends, E. 2000. Introduction to Markov chains: with special emphasis on rapid mixing. Advanced Lecture Notes in Mathematics ed. Vieweg Verlag: Free University of Berlin.

Berger, L. R. 1981. "A necessary and sufficient condition for reaching a consensus using DeGroot's method.". Journal of the American Statistical Association 76 (374): 415-418.

Brown, R. 1988. Group processes: Dynamics within and between groups. Basil Blackwell.

Buechel, B., T. Hellmann, and S. Klößner. 2013. "Opinion Dynamics under Conformity”. Working Paper, Institute of Mathematical Economics, Bielefeld University.

Buechel, B., T. Hellmann, and M. Pichler. 2012. "The Dynamics of Continuous Cultural Traits in Social Networks". Working Paper Bielefeld University, Institute of Mathematical Economics (457).

Butera, F., G. Mugny, P. Legrenzi, and J. a. Perez. 1996. "Majority and minority influence, task representation and inductive reasoning". British Journal of Social Psychology 35:123-136.

Crano, W. D., and V. Seyranian. 2007. "Majority and minority influence". Social and Personality Psychology Compass 1:572-589.

DeGroot, M. H. 1974. "Reaching a Consensus". Journal of the American Statistical Association 69 (345): $118-121$.

Ellero, A., G. Fasano, and A. Sorato. 2013. "Stochastic model of agent interaction with opinion leaders". Physical Review E 87 (4): 042806.

Festinger, L. 1954. "A theory of social comparison processes". Human relations 7 (2): 117-140.

Galam, S. 2003. "Modelling rumors: the no plane Pentagon French hoax case". Physica A: Statistical Mechanics and Its Applications 320:571-580.

Galam, S. 2012. Sociophysics, A Physicist's Modeling of Psycho-political Phenomena. Understanding Complex Systems. Springer.

Galam, S., Y. Gefen, and Y. Shapir. 1982. "Sociophysics: A new approach of sociological collective behaviour. 1. Mean-behaviour description of a strike". The Journal of Mathematical Sociology 9 (1): $1-13$.

Galam, S., and S. Moscovici. 1991. "Towards a theory of collective phenomena: Consensus and attitude changes in groups". European Journal of Social Psychology 21 (1): 49-74.

Hare, I. P., and R. F. Bales. 1963. "Seating position and small group interaction". Sociometry 26:480-486.

Hegselmann, R., S. König, S. Kurz, C. Niemann, and J. Rambau. 2014. "Optimal opinion control: The campaign problem". Available at SSRN 2516866.

Hegselmann, R., and U. Krause. 2002. "Opinion dynamics and bounded confidence models, analysis, and simulation". Journal of Artificial Societies and Social Simulation 5 (3): 1-32.

Ibe, O. C. 2009. Markov processes for stochastic modeling. London, UK: Elsevier Academic Press.

Maass, A., and R. D. Clark. 1984. "Hidden impact of minorities: Fifteen years of minority influence research". Psychological Bulletin 95 (3): 428-450.

Merlone, U., and D. Radi. 2014. "Reaching consensus on rumors". Physica A: Statistical Mechanics and its Applications 406:260-271.

Merlone, U. and Romano, A. and Radi, D. "Opinion Dynamics on Networks". Working paper.

Moscovici, S., and E. Lage. 1976. "Studies in social influence III: Majority versus minority influence in a group". European Journal of Social Psychology 6 (2): 149-174.

Moscovici, S., E. Lage, and M. Naffrechoux. 1969. "Influence of a consistent minority on the responses of a majority in a color perception task". Sociometry 32 (4): 365-380.

Moscovici, S., and B. Personnaz. 1980. "Studies in social influence". Journal of Experimental Social Psychology 16:270-282. 
Mugny, G. 1975. "Negotiations, image of the other and the process of minority influence". European Journal of Social Psychology 5 (2): 209-228.

Mugny, G., and S. Papastamou. 1980. "When rigidity does not fail: Individualization and psychologization as resistances to the diffusion of minority innovations". European Journal of Social Psychology 10 (1): 43-61.

Mugny, G., and S. Papastamou. 1982. The power of minorities, Volume 31. Academic Press.

Nemeth, C., and J. Wachtler. 1974. "Creating the perceptions of consistency and confidence: A necessary condition for minority influence". Sociometry 37 (4): 529-540.

Nyczka, P., and K. Sznajd-Weron. 2013. "Anticonformity or Independence?-Insights from Statistical Physics". Journal of Statistical Physics 151 (1-2): 174-202.

Paichler, G. 1976. "Norms and attitude change I: Polarization and styles of behaviour". European Journal of Social Psychology 6 (4): 405-427.

Sargent, R. G. 1984. "A Tutorial on Verification and Validation of Simulation Models". In Proceedings of the 1984 Winter Simulation Conference, edited by S. Sheppard, U. Pooch, and D. Pegden, 115-121. IEEE Press.

Shuper, P., and R. M. Sorrentino. 2004. "Minority versus majority influence and uncertainty orientation: processing persuasive messages on the basis of situational expectancies". The Journal of social psychology 144 (2): 127-147.

Sommer, R. 1961. "Leadership and group geography". Sociometry 24:99-110.

Stauffer, D. 2012. "A biased review of sociophysics". Journal of Statistical Physics 151 (1-2): 9-20.

Strodtbeck, F. L., and L. H. Hook. 1961. "The social dimensions of a twelve man jury table". Sociometry 24:397-415.

West, B. J., M. Turalska, and P. Grigolini. 2014. Networks of Echoes. Springer.

Wood, W., S. Lundgren, J. A. Ouellette, S. Busceme, and T. Blackstone. 1994. "Minority influence: a meta-analytic review of social influence processes". Psychological bulletin 115 (3): 323-345.

\section{AUTHOR BIOGRAPHIES}

UGO MERLONE (Ph.D. in Applied Mathematics, University of Trieste, Italy) is Associate Professor at the Psychology Department, University of Torino, Italy. His main area of interest is the modeling of human behavior and organizations. On these topics he has published on journals such as European Journal of Operational Research, Physica A, Journal of Economic Behavior \& Organization, Journal of Mathematical Sociology, International Game Theory Review, Organization Science, Journal of Artificial Societies and Social Simulation, Mathematics and Computers in Simulation, and Communications in Nonlinear Science and Numerical Simulation. Further details can be found on his homepage www.ugomerlone.net. His email address is ugo.merlone@unito.it.

DAVIDE RADI (Ph.D. in Economics, Applied Mathematics and Operational Research, University of Bergamo, Italy) is Post-Doc at the Department of Management, Polytechnic University of Marche, Italy. His research interests include game theory and modeling of human behavior. His email address is d.radi@ univpm.it.

ANGELO ROMANO is a PhD student at Department of Psychology, Torino, Italy. His theoretic research focuses on the overlapping area of social psychology and simulation. His applied research includes cooperation, social dilemmas and opinion dynamics. His email address is a.romano@unito.it. 